Rev. Elev. Méd. vét. Pays trop., 1975, 28 (3) : 287-295

\title{
Etude sérologique comparative des principales souches de virus aphteux isolés en Ethiopie de 1969 à 1974
}

\author{
par J. L. MARTEL (*) \\ (avec la collaboration technique de C. GALLON) (*)
}

\begin{abstract}
RESUME
Dans une note précédente, l'inventaire des types de virus aphteux isolés en Ethiopie a été présenté. Mais la notion de type étant insuffisante, nous avons cherché à mettre en évidence l'existence éventuelle de variantes au sein de chaque type $\mathrm{O}, \mathrm{A}$ et $\mathrm{C}$ des souches éthiopiennes dont nous disposons. Nous avons utilisé une technique sérologique de fixation du complément quantitative de semi-précision. Compte tenu des limites de cette méthode, un premier tri sérologique des souches éthiopiennes a pu être fait. Ce tri sérologique constitue une étape préliminaire indispensable pour orienter les études immunologiques ultérieures.
\end{abstract}

\section{INTRODUCTION}

Dans une note précédente (8) nous avons précisé que seuls les types $\mathrm{O}, \mathrm{A}$ et $\mathrm{C}$ de virus aphteux ont été isolés à ce jour en Ethiopie.

Mais l'étude épidémiologique complète de la fièvre aphteuse dans une région donnée ne saurait se contenter d'un simple inventaire des types de virus isolés. En effet, dans le cadre d'un même type, les souches de virus aphteux montrent quelques différences dans leur comportement antigénique $(11,12)$.

Actuellement les méthodes de sérologie et d'immunologie quantitatives permettent de distinguer un nombre important de variantes à l'intérieur d'un même type $(2,3,4,5,6,7$, $9,10)$. Cette distinction a un intérêt pratique majeur: elle constitue l'élément essentiel du choix des souches utilisées pour la préparation et le contrôle des vaccins antiaphteux.

(*) Imperial Veterinary Institute et Mission Vétérinaire Françaiss en Ethiopie, P.O.B. 19, Debré-Zeit, Ethiopie.
Une telle étude épizootologique a été commencée par une équipe de vétérinaires du Laboratoire Roger Bellon (1) avec les premières souches de type $\mathrm{O}$ et $\mathrm{A}$ isolées en Ethiopie jusqu'au premier semestre 1971. Cette première étude, assez avancée pour le type A, n'avait été qu'ébauchée pour le type $O$, et l'étude du type $C$ n'avait pas pu être abordée faute d'isolement de virus de ce type à l'époque.

Nous avons repris ce travail en élargissant l'éventail des souches, mais en nous limitant par contre, pour des raisons matérielles, à l'aspect sérologique.

\section{MATERIEL ET METHODE}

\section{A) Principe}

Nous avons titré, par fixation du complément, les anticorps antiaphteux préparés par hyperimmunisation de cobayes avec les diverses souches de virus aphteux à notre disposition. 
Ces titrages ont été réalisés en présence d'une part des antigènes correspondants (titre homologue) et d'autre part en présence des autres antigènes de même type (titres hétérologues).

Les rapports entre les titres homologues et les titres hétérologues caractérisent les relations sérologiques entre les souches.

\section{B) Les éléments de la réaction sérologique}

1. Le sérum hémolytique de lapin antihématies de moutons est celui du commerce.

2. Les hématies de moutons proviennent des moutons locaux. Elles sont utilisées après avoir été lavées trois fois dans du tampon phosphate isotonique.

3. Le complément utilisé est du complément lyophilisé du commerce, que l'on reconstitue avec du tampon acide borique-acétate.

4. Les sérums hyperimmuns ont été préparés sur cobayes selon la technique décrite dans la publication précédente (8).

Les sérums hyperimmuns correspondant aux souches européennes nous ont été fournis par le Laboratoire Roger Bellon.

5. Les antigènes sont préparés sur cultures cellulaires en flacons roulants de la lignée IB RS 2, à partir des souches de virus isolés en
Ethiopie. La liste des souches retenues pour cette étude est présentée dans le tableau $\mathbf{n}^{\circ} \mathrm{I}$. Nous avons fait ce choix en fonction de critères chronologiques et géographiques.

Le virus est utilisé si possible dès le troisième passage sur culture cellulaire pour éviter au maximum toute variation par rapport au virus sauvage. La récolte est clarifiée par centrifugation, mais le virus n'est pas inactivé. Dans la plupart des cas, les antigènes ainsi préparés fixent complètement le complément au moins jusqu'au $1 / 8$ lorsqu'ils sont mis en présence du sérum homologue.

Les antigènes d'origine européenne nous ont été fournis par le Laboratoire Roger Bellon après inactivation.

\section{C) La réaction sérologique}

\section{Choix de la technique}

La réaction de fixation du complément utilisée est du type Kolmer à 100 p. 100 d'hémolyse, manuelle, en tube (3).

Cette technique est quantitative : le complément est utilisé à la dilution limite donnant 100 p. 100 d'hémolyse lors du titrage en présence de l'antigène seul [c'est-à-dire une unité hémolytique au lieu de deux comme dans la technique qualitative de typage (8)].

TABLEAU N $N^{\circ}$ I - Liste des souches éthiopiennes étudiêes.

\begin{tabular}{|c|c|c|c|c|}
\hline Type & Lieu d'isolement & Province & Epoque du foyer & $\mathrm{N}^{0}$ référence ${ }^{+}$ \\
\hline \multirow[t]{6}{*}{0} & Ghinda & Erythrée & 1969 & 22 \\
\hline & Shola & Centre Shoa & 1970 & 27 \\
\hline & Fiche & Nord Shoa & 1970 & 38 \\
\hline & Awasa & Nord Sidamo & mars 1972 & 45 \\
\hline & Lekempti & Wo1lega & mai 1972 & 50 \\
\hline & Holeta & Centre Shoa & décembre 1972 & 76 \\
\hline \multirow[t]{5}{*}{$A$} & Keren & Erythrëe & 1969 & 23 \\
\hline & Asmara & Erythrêe & 1970 & 31 \\
\hline & Adidis & Shoa & 1970 & 33 \\
\hline & $L \in i$ & Sidamo & 1970 & 37 \\
\hline & Koka & Shoa & fëvrier 1974 & 99 \\
\hline \multirow[t]{4}{*}{$\mathrm{C}$} & Shashemene & Sud Shoa & août 1971 & 40 \\
\hline & Debre Tsigue & Nord Shoa & décembre 1971 & 43 \\
\hline & Asella & Arussi & juillet 1972 & 54 \\
\hline & Sendafa & Nord Shoa & septembre 1972 & 57 \\
\hline
\end{tabular}

+ : les $\mathrm{N}^{0}$ de référence sont les mêmes que ceux utilisés dans la note précédente. On pourra se reporter à la publication précédente pour situer les lieux d'isolement sur la carte de répartition des typea de virus aphteux en Ethiopie. 
Nous devons reconnaître qu'il existe d'autres techniques de fixation du complément quantitatives plus précises (lecture de l'hémolyse 50 p. 100 , automatisation) que celle que nous avons choisie. Nous avons retenu toutefois cette technique de semi-précision car elle correspond à nos possibilités de travail local.

\section{Réalisation des titrages \\ des sérums hyperimmuns}

On réalise des titrages en échiquier, en mettant en présence chaque sérum hyperimmun dilué selon une progression géométrique de raison 1,5 avec des séries d'antigène dilué en progression géométrique de raison 2 .

Le titre du sérum est l'inverse de la dilution limite du sérum fixant totalement le complément dans les conditions optimales d'association avec l'antigène.

\section{D) Calcul de la parenté entre les souches de même type}

Le titrage du sérum hyperimmun $a$, préparé à partir d'une souche $a$ est réalisé d'une part vis-à-vis de l'antigène $a$ (titre homologue) et d'autre part vis-à-vis d'un antigène $b$ (titre hétérologue). Ces deux titres permettent d'établir le rapport :

Titre hétérologue du sérum anti $a$

r $(a)=\frac{\text { contre l'antigène } b}{\text { Titre homologue du sérum anti } a}$

De même, le double titrage du sérum hyperimmun $b$ vis-à-vis des antigènes $b$ et $a$ donne un titre homologue et un titre hétérologue permettant d'établir le rapport :

Titre hétérologue du sérum anti $b$

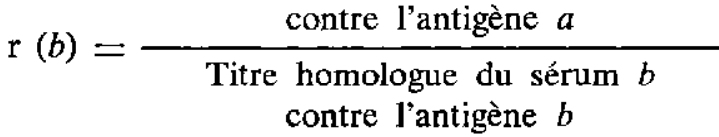

Les rapports $\mathbf{I}(a)$ et $\mathbf{r}(b)$ représentent les relations sérologiques unilatérales entre les souches $a$ et $b$ : elles sont comprises entre 0 et 1 . Plus ces rapports sont proches de 1 , plus les souches sont semblables. Plus ces rapports sont éloignés de l'unité, plus elles sont différentes.

Ces rapports servent à préciser la parenté sérologique bilatérale $R$, exprimée en pourcentage et calculée selon la formule :

$$
\mathrm{R}=100 \sqrt{\mathrm{r}(a) \cdot \mathrm{r}(b)}
$$

$\mathrm{R}=100$ p. 100 représente l'identité sérologique bilatérale complète. Par contre, dans l'état actuel de nos connaissances, il est difficile de situer la limite à partir de laquelle la notion de variante apparaît. DAVIE (4) pense qu'on ne peut affirmer une différence certaine entre deux souches que lorsque le rapport $\mathrm{r}$ est inférieur à 0,50 .

\section{RESULTATS}

Les résultats des titrages des sérums hyperimmuns anti $\mathrm{O}, \mathrm{A}$ et $\mathrm{C}$ figurent respectivement dans les tableaux $n^{\circ *}$ II, $V$ et VII. Sur une même ligne horizontale, on peut lire les titres d'un même sérum vis-à-vis des divers antigènes essayés. Nous avons encadré les titres homologues.

Nous n'avons présenté que les relations sérologiques unilatérales $r$ des deux souches $O$ Ghinda et $\mathrm{O}$ Fiche vis-à-vis des autres souches de type $O$ (tableau $n^{0}$ III). En effet, nous n'avons pas pu calculer les parentés $R$ pour ces deux souches.

Pour les autres souches, nous avons indiqué directement les parentés sérologiques bilatérales $R$ que nous avons regroupées dans les tableaux $n^{\text {os }}$ IV, VI et VIII respectivement pour les souches de type $\mathrm{O}$, $\mathrm{A}$ et $\mathrm{C}$.

TABLEAU $\mathrm{N}^{\circ} \mathrm{II}-\mathrm{Titres}$ des sêrums hyperimmuns de type 0

\begin{tabular}{|c|c|c|c|c|c|c|c|c|}
\hline & Antigēnes & Laus anne & Ghinda & Shola & Fiche & Awasa & Lekempti & Haleta \\
\hline \multirow{7}{*}{ 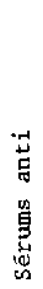 } & o Lausanne & 113 & 113 & 94 & 113 & 50 & 113 & 113 \\
\hline & O Ghinda & 20 & 50 & 44 & 22 & 27 & 33 & 27 \\
\hline & o Shola & 65 & 75 & 140 & 170 & 65 & 113 & 75 \\
\hline & o Fiche & 15 & 33 & 44 & 33 & 22 & 33 & 27 \\
\hline & O Awasa & 50 & 75 & 75 & 75 & 75 & 75 & 50 \\
\hline & O Lekempti & 75 & 113 & 113 & 113 & 50 & 170 & 113 \\
\hline & O Holeta & 113 & 113 & 113 & 94 & 65 & 113 & 113 \\
\hline
\end{tabular}

Les titres homologues sont encadrés. 
TABL. $N^{\circ}$ III-Relations sêrologiques unilatêrales entre les souches o Ghinda et 0 Fiche d'une part et les autres souches de type 0.

\begin{tabular}{|c|c|c|c|c|c|}
\hline Essai des sêrums antí 0 & Lausanne & Shola & Awasa & Lekempti & Holeta \\
\hline $\begin{array}{l}\text { contre l'antigène } \\
\text { Ghinda }\end{array}$ & 1 & 0,54 & 1 & 0,66 & 1 \\
\hline $\begin{array}{l}\text { contre l'antigẽne } \\
\text { Fiche }\end{array}$ & 1 & 1 & 1 & 0,66 & 0,83 \\
\hline
\end{tabular}

TABL. N ${ }^{\circ}$ IV - Parentés sérologiques bilatérales entre les souches de type 0.

\begin{tabular}{|c|c|c|c|c|c|}
\hline & O Lausanne & O Shola & O Awasa & o Lekempti & O Holeta \\
\hline o Lausanne & 100 & 62 & 54 & 66 & 100 \\
\hline O Shola & 62 & 100 & 68 & 73 & 73 \\
\hline O Awasa & 54 & 68 & 100 & 53 & 6] \\
\hline o Lekempti & 66 & 73 & 53 & 100 & 81 \\
\hline O Holeta & 100 & 73 & 61 & 81 & 100 \\
\hline
\end{tabular}

TABLEAU $\mathrm{N}^{\mathrm{V}} \mathrm{V}-$ Titre des sêrums hyperimmuns de type A.

\begin{tabular}{|c|c|c|c|c|c|c|c|}
\hline \multicolumn{2}{|c|}{ Antigènes } & \multirow{2}{*}{$\frac{A 5}{\left(256^{+}\right)}$} & \multirow{2}{*}{ A Keren } & \multirow{2}{*}{$\frac{\text { A Asmara }}{75}$} & \multirow{2}{*}{$\frac{\text { A Addis }}{75}$} & \multirow{2}{*}{$\begin{array}{c}\text { A Lei } \\
33\end{array}$} & \multirow{2}{*}{$\frac{\text { A Koka }}{75}$} \\
\hline & A 5 & & & & & & \\
\hline & A Keren & - & 256 & 256 & 170 & 113 & 170 \\
\hline ' & A Asmara & - & 384 & 320 & 213 & 113 & 170 \\
\hline 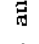 & A Addis & - & 140 & 113 & 256 & 50 & 256 \\
\hline 罟 & A Lei & - & 256 & 170 & 256 & 256 & 256 \\
\hline 忽 & A Koka & - & 50 & 75 & 113 & 75 & 113 \\
\hline
\end{tabular}

+ : titre indiqué par le producteur.

TABLEAU N $N^{\circ} \mathrm{I}$ - Parentés sérologiques bilatërales entre les souches de type A.

\begin{tabular}{|l|ccccc|}
\hline & A Keren & A Asmara & A Lei & A Addis & A Koka \\
\hline A Keren & 100 & 100 & 66 & 60 & 54 \\
A Asmara & 100 & 48 & 48 & 54 & 59 \\
A Le1 & 66 & 100 & 43 & 81 \\
A Addis & 60 & 59 & 43 & 100 & 100 \\
A Koka & 54 & & 100 & 100 \\
\hline
\end{tabular}

TABLEAU $\mathbb{N}^{\circ}$ VII - Titres des sêrums hyperimmuns de type $\mathrm{C}$.

\begin{tabular}{|c|c|c|c|c|c|c|}
\hline & Antıgènes & Europe & Shashemene & Debre Tsigue & Ase11a & Sendafa \\
\hline \multirow{5}{*}{ 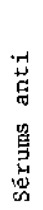 } & Europe & 170 & 113 & 113 & 170 & 140 \\
\hline & Shashemene & 75 & 170 & 170 & 170 & 113 \\
\hline & Debre Tsigue & 170 & 256 & 256 & 256 & 256 \\
\hline & Asella & 50 & 113 & 113 & 213 & 113 \\
\hline & Sendafa & 170 & 256 & 256 & 256 & 256 \\
\hline
\end{tabular}


TABLEAU N*VIII - Parentës sérologiques bilatêrales entre les souches de type C.

\begin{tabular}{|c|c|c|c|c|c|}
\hline & Europe & Shashetrene & Debre Tsigue & Asella & Sendafa \\
\hline C Europe & 100 & 54 & 66 & 66 & 73 \\
\hline C Shashemene & 54 & 100 & 100 & 100 & 81 \\
\hline C Debre Tsigue & 66 & 100 & 100 & 100 & 100 \\
\hline C Ase11a & 66 & 100 & 100 & 100 & 100 \\
\hline C Sendafa & 73 & 81 & 100 & 100 & 100 \\
\hline
\end{tabular}

\section{DISCUSSION}

\section{Souches de type 0}

Lorsque l'on considère les titres des sérums hyperimmuns de type $O$, on constate que les titres des sérums anti $O$ Ghinda et $O$ Fiche sont nettement insuffisants. Aussi n'avons-nous pas retenu ces deux derniers sérums pour les calculs ultérieurs.

Par contre, nous avons conservé les antigènes $O$ Ghinda et $O$ Fiche pour titrer les sérums hétérologues et calculer les relations unilatérales $r$. Ces rapports $r$ (tableau $\mathrm{n}^{0}$ III) étant au moins égaux à 0,54 , on peut penser que les souches $O$ Ghinda et $O$ Fiche ne sont pas très éloignées des autres souches de type $O$.
Les parentés sérologiques bilatérales calculées pour les autres souches sont regroupées dans le tableau $\mathbf{n}^{\circ} \mathrm{IV}$; les résultats concernant les souches $O$ éthiopiennes entre elles sont encadrés. On note en effet une assez grande homogénéité entre ces souches, surtout si l'on ne tient pas compte de la souche O Awasa dont les réactifs se sont avérés médiocres.

Parmi les souches de type $O$, O Holéta présente les plus forts pourcentages de parenté avec l'ensemble des autres souches, tant éthiopiennes que la souche $O$ Lausanne. De plus, il faut préciser que cette souche fut responsable d'une rupture d'immunité : elle a été isolée sur des bovins laitiers régulièrement vaccinés contre la fièvre aphteuse avec un vaccin trivalent $O$,

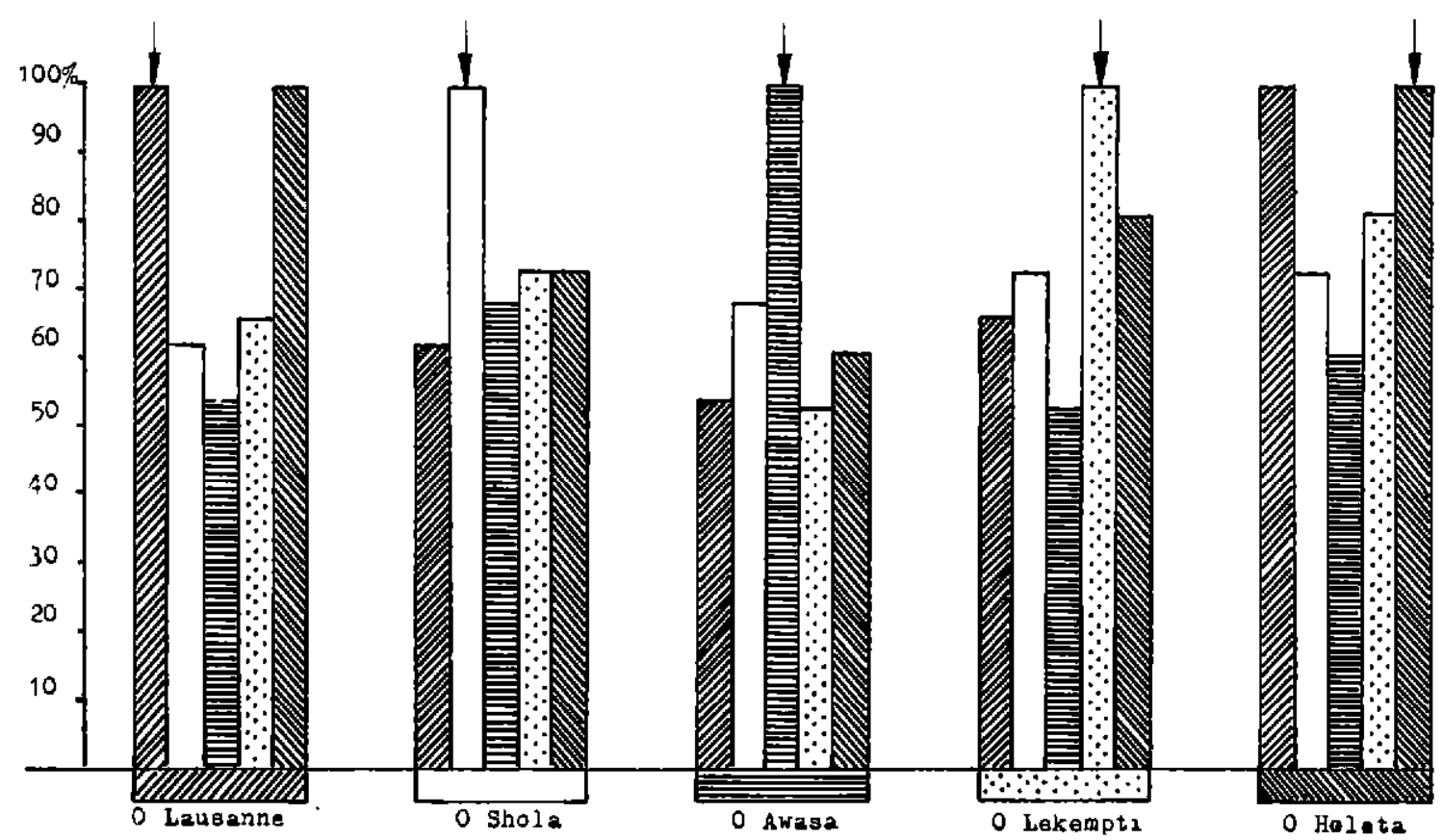

Nous avons indiqué en abscisse les sérums hyperimmuns de référence. En ordonnée se trouvent les parentés sćrologiques bilatérales $R$ exprimées en pourcentage. Chaque flèche indique la souche homologue de chaque sérum de référence : dans ce cas la parenté $R$ atteint évidemment la valeur 100 p. 100 . Ce diagramme représente les différents pourcentages $R$ obtenus pour chaque sérum quand on essaie les différentes souches de type $O$. 
A, C préparé au Kenya à partir de souches isolées en Afrique Orientale (la valence $\mathrm{O}$ est d'origine Kényanne: O K120/ 64). Ces données suggèrent que la souche $O$ Holéta pourrait bien être une dominante parmi les souches éthiopiennes et même parmi les souches d'Afrique Orientale.

Enfin, la souche européenne $O$ Lausanne n'apparaît pas trop éloignée de l'ensemble des souches $\mathrm{O}$ éthiopiennes: le pourcentage de parenté le plus faible $(54$ p. 100$)$ se situe audessus du seuil des 50 p. 100 et il s'observe avec la souche O Awasa au sujet de laquelle nous avons déjà fait quelques réserves sur la qualité.

\section{Souches de type $A$}

Les titres des sérums anti A sont regroupés dans le tableau $n^{\circ} \mathrm{V}$.

L'antigène inactivé A 5 (A Allier) dont nous disposions s'est révélé de très mauvaise qualité lorsque nous l'avons utilisé après un trop long stockage à $+4^{\circ} \mathrm{C}$. Nous n'avons pas retenu les titres des sérums vis-à-vis de cet antigène.
Par contre la conservation, au congélateur, du sérum hyperimmun anti A 5, sous forme lyophilisée, ne pose aucun problème: ce sérum est utilisable avec quelques réserves toutefois. En effet, n'ayant pas pu faire le titrage homologue de ce sérum avec notre système réactionnel, puisque l'antigène A 5 était inutilisable, les titres hétérologues ne sont pas directement comparables au titre homologue annoncé par le producteur du sérum. Néanmoins, les titres hétérologues étant relativement faibles, on peut penser que la souche A 5 est assez éloignée sérologiquement des souches éthiopiennes.

Le tableau $\mathrm{n}^{0}$ VI présente les résultats du calcul des parentés sérologiques bilatérales entre les souches éthiopiennes de type A. Nous avons encadré les résultats qui suggèrent une très grande parenté entre certaines souches. On met ainsi en évidence deux ensembles distincts :

1. Il y a pratiquement identité sérologique entre les deux souches érythréennes A Kéren, isolée en 1969, et A Asmara, isolée en 1970.

2. On retrouve la même identité entre deux souches isolées dans le Shoa: A Addis, isolée en 1970, et A Koka isolée en 1974. Par ailleurs,

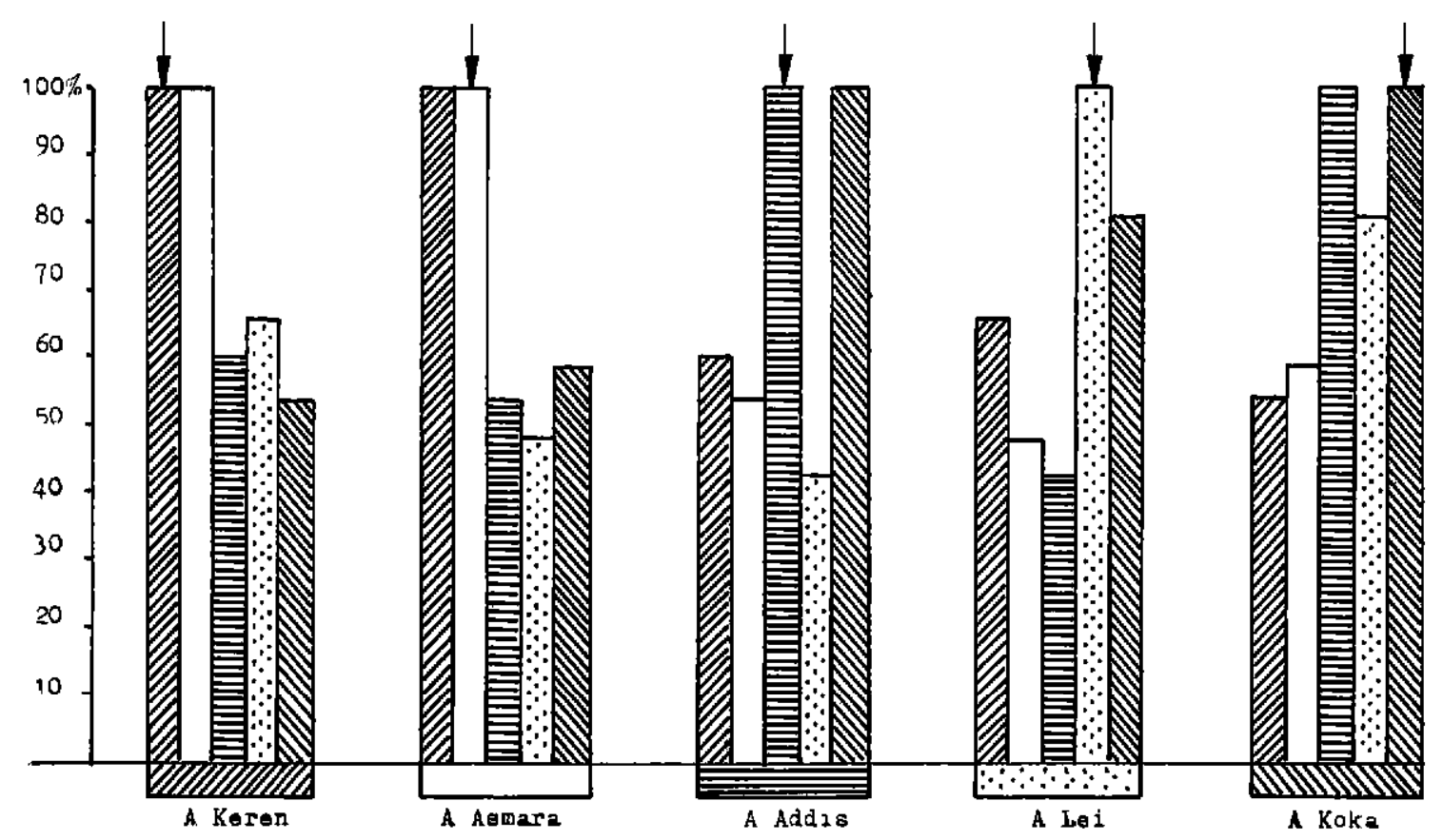

Nous avons indiqué en abscisse les sérums de référence. En ordonnéz se trouvent les parentés sérologiques bilatérales $R$ exprimées en pourcentage. Chaque flèche indique la souche homologue de chaque sérum de référence : dans ce cas le pourcentage $R$ atteint évidemment la valeur 100 p. 100 . Ce diagramme représente les différents pourcentages $R$ obtenus pour chaque sérum quand on essaie les différentes souches de type $A$. 
la souche A Lei, isolée dans l'extrême sud de l'Ethiopie, semble assez proche de la souche A Koka.

Il semble donc que du point de vue des virus aphteux de type A, il faille distinguer en Ethiopie deux zones épidémiologiques: une zone nord avec les souches erythréennes et une zone centre-sud avec la souche A Koka comme souche de référence $(*)$. Cela correspond d'ailleurs à deux régions d'élevage géographiquement isolées.

\section{Souches de type $C$}

Si l'on considère les titres des sérums hyperimmuns de type $C$ (tableau $n^{\circ}$ VII), tous ces sérums se révèlent satisfaisants.

Le calcul des pourcentages $R$ de parentés sérologiques bilatérales (tableau $n^{\circ}$ VIII) fait

(*) Depuis la rédaction de ce texte, l'épizootie de type A s'est largement étendue sur l'ensemble de la province du Shoa. Une nouvelle souche de ce type, isolée en novembre 1974, a retenu notre attention car elle est responsable d'une rupture d'immunité. L'étude sérologique et immunologique de cette souche est en cours. Ce fait montre le caractère provisoire de nos conclusions et la nécessité de surveiller en permanence l'évolution des souches. apparaître la très grande homogénéité des souches éthiopiennes de type C. Ceci n'est peutêtre que le reflet de l'unité épidémiologique observée sur le terrain: les foyers de type C que nous avons étudiés, tout en étant nombreux et sévères, sont assez limités géographiquement.

La souche $\mathrm{C}$ européenne diffère peu des souches de type $\mathrm{C}$ éthiopiennes.

\section{CONCLUSIONS}

Compte tenu des réserves avancées quant à la précision de la technique sérologique utilisée, le présent travail permet de faire un premier tri des souches de virus aphteux isolés en Ethiopie depuis 1969.

L'ensemble des souches de type O éthiopiennes contrôlées apparaît assez homogène et peu éloigné de la souche $\mathrm{O}$ Lausanne.

Parmi les souches de type $O$, la souche $O$ Holéta semble être sérologiquement la plus complète. L'étude immunologique de cette souche mérite d'être entreprise.

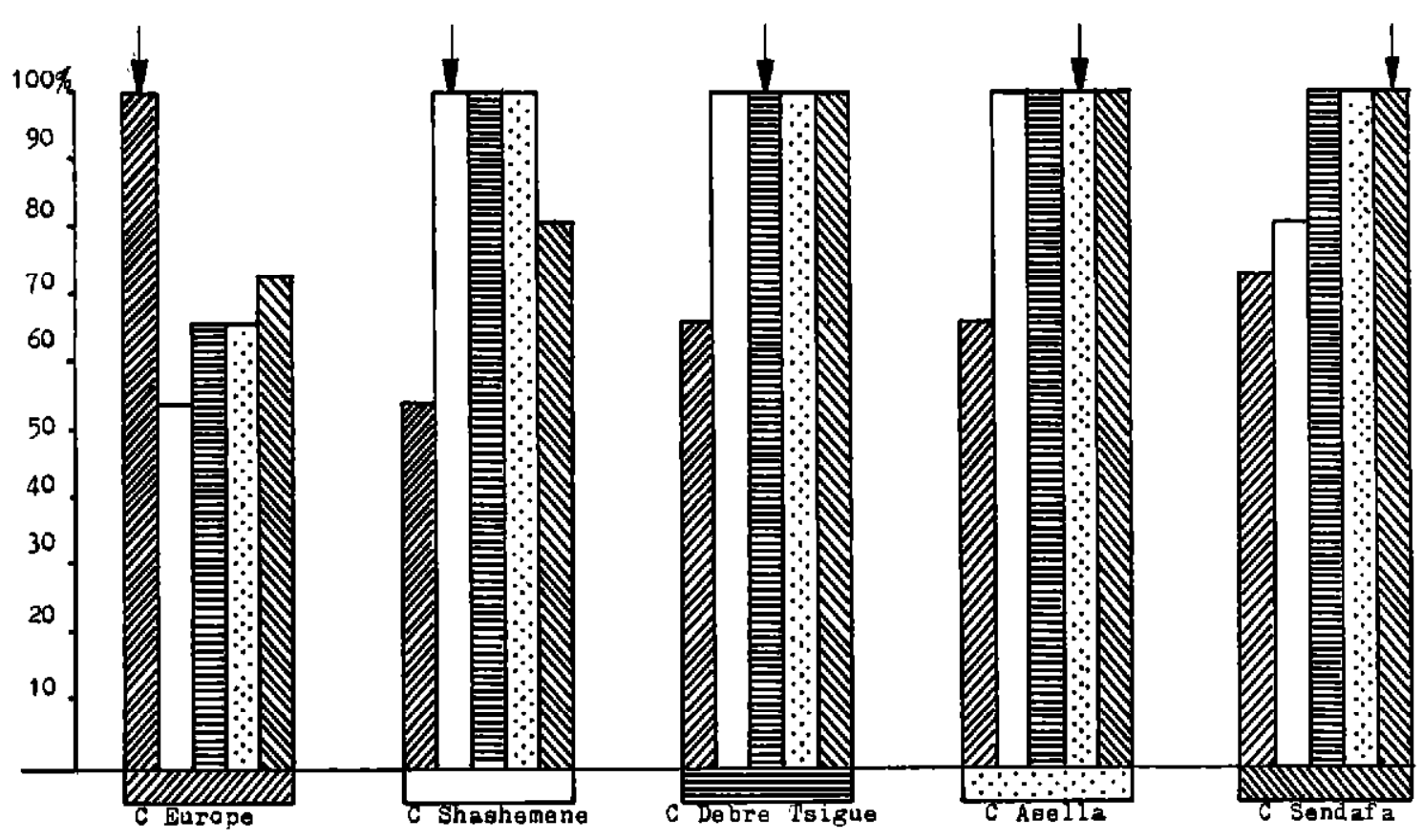

Nous avons indiqué en abscisse les sérums de référence. En ordonnée se trouvent les parentés sérologiques bilatérales $R$ exprimées en pourcentage. Chaque flèche indique la souche homologue de chaque sérum de référence : dans ce cas le pourcentage $R$ atteint évidemment la valeur 100 p. 100 . Ce diagramme représente les différents pourcentages $R$ obtenus pour chaque sérum quand on essaie les différentes souchss de type $C$. 
En première approximation, toutes les souches de type A éthiopiennes étudiées sembleraient sérologiquement distinctes de la souche A 5 européenne.

Les études immunologiques des souches $\mathrm{A}$ éthiopiennes devront tenir compte des deux zones épidémiologiques individualisées par la sérologie.

Enfin, les souches de type $\mathrm{C}$ paraissent très proches les unes des autres. Cette homogénéité reflète probablement l'unité épidémiologique observée sur le terrain.

L'intérêt d'une telle étude n'est pas simplement spéculatif. Elle constitue une étape préliminaire indispensable dans le tri des souches éthiopiennes. Il s'agit en pratique de choisir dans la collection des virus aphteux isolés en Ethiopie :
1. des souches d'épreuve pour le contrôle d'efficacité des vaccins utilisés en Ethiopie (qu'ils soient importés ou produits localement),

2. des souches vaccinales pour la production éventuelle de vaccins locaux.

\section{Remerciements}

Nous remercions le Laboratoire Roger Bellon, Usine de la Croisette (Directeur : M. GIRAUD) à Villaines-les-Rochers par Azay-leRideau - France, qui nous a fait parvenir les antigènes inactivés et les sérums hyperimmuns correspondant aux souches européennes.

Nous tenons à remercier également les Docteurs Vétérinaires J.P. BERSON et X. COLSON qui ont isolé, en 1969 et 1970, les premières souches éthiopiennes étudiées ici et qui ont préparé les sérums hyperimmuns à partir de ces souches.

\section{SUMMARY}

Comparative serological study of principal strains of foot-and-mouth disease virus isolated in Ethiopia from 1969 to 1974

In a previous work, the foot-and-mouth disease virus types isolated in Ethiopia were inventoried.

The type notion being insufficient, the author investigated the possible existence of variants in each $\mathrm{O}, \mathrm{A}$ and $\mathrm{C}$ type of available ethiopian strains.

A serological test of semi-accurate quantitative complement fixation was used and first sorted out serologically ethiopian strains.

This serological sorting constitutes a previous stage indispensable for directing further immunological studies.

\section{RESUMEN}

Estudio serologico comparativo de las principales cepas de virus aftoso aisladas en Etiopia de 1969 a 1974

En otro artículo, se hizo el inventario de los tipos de virus aftoso aislados en Etiopia.

Pero siendo insuficiente la hoción de tipo, se ha tratado de poner en evidencia la existencia eventual de variantes para cada tipo $\mathrm{O}, \mathrm{A}$ y $\mathrm{C}$ de las cepas etiopianas de que se dispone.

Se utilizó una tecnica serologica de fijación del complemento cuantitativa de semiprecisión.

Habida cuenta de los límites de dicho método, se pudo efectuar una primera tria serologica de las cepas etiopianas.

Esta tria serologica constituye una etapa preliminar indispensable para orientar los estudios inmunologicos ulteriores. 


\section{BIBLIOGRAPHIE}

1. BERSON (J.P.), COLSON (X.), FIKRE (J.), VIGIER (M.), ASSEFA (W. G.), GUERCHE (J.), BLANC (R.), PRUNET (P.), Etude épizootologique de la fièvre aphteuse en Ethiopie (1969. 1971). Bull. off. int. Epiz., 1972, 77 (3-4): 395620.

2. BROOKSBY (J. B.). The technique of complement fixation in foot and mouth disease research. London, Her Majesty's Stationnery Office, 1952. (Agricultural Research Council Report Series no 12.)

3. CAMAND (R.). Etude sérologique des types et des variantes du virus aphteux par la réaction de fixation du complément. Thèse Doct. Méd. vét., Lyon, 1953, no 3 .

4. DAVIE (J.). The classification of subtype variants of the virus of foot and mouth disease. Bull. off. int. Epiz., 1962, 57:962.

5. DAVIE (J.). A complement fixation technique for the quantitative measurement of antigenic differences between strains of the foot and mouth disease virus. J. Hyg. Camb., 1964, 62: 401-411.

6. JOUBERT (L), MACKOWIAK (C.). La fièyre aphteuse. Volume I: Le virus aphteux. Paris, Expansion Scientifique Française. 1968.

7. MACKOWIAK (C.), FONTAINE (J.), ROUMIANTZEFF (M.). Types, sous-types et varian- tes du vìrus aphteux. Etude des variantes. 19th. Symp. intern., Section Permanente de Standardisation Microbiologique "Fièvre Aphteuse" variantes et immunité. Lyon, 14 juillet 1967. Volume 8, Bâle, Karger, pp. 13-64.

8. MARTEL (J.L.). La fièvre aphteuse en Ethiopie. Distribution des sérotypes de virus aphteux. Rev. Elev. Mêd. vét. Pays trop., 1974, 27 (2) : 169-175.

9. ROUMIANTZEFF (M.), STELLMANN (C.), DUBOUCLARD (C.). Technique de fixation quantitative du complément appliquée à l'étude du virus de la fièvre aphteuse. Bull. Soc. Sci. vét. Méd. Comp., Lyon, 1965, $67:$ 243-269.

10. ROUMIANTZEFF (M.), DUBOUCLARD (C.), FONTAINE (J.), GILBERT (H.). Méthodes sérologiques utilisées pour l'étude des variantes du virus aphteux. Bull. Soc, Sci. vét. Méd. comp., Lyon, 1966, 68: 41-54.

11. TRAUB (E.), MOLHMANN (E.). Typification de la fièvre aphteuse par la réaction de fixation du complément. Zentralbt. Bakt. I. Orig., 1953, 150 : 289-310.

12. VALLEE (H.). Sur la pluralité du virus aphteux. Bull. Off. int. Epiz., 1928, 1: 500.

13. VILLON (A.). Epizootologie de la fièvre aphteuse en Ethiopie. Identification des types de virus. Thèse Doct. Méd. vét., Lyon, 1973, nº 51. 